\title{
Characterisation of the impact response of energetic materials: observation of a low-level reaction in 2,6-diamino-3,5-dinitropyrazine-1-oxide (LLM-105)
}

\author{
David M. Williamson, Sue Gymer, Nicholas E. Taylor, Stephen M. Walley* and Andrew P. Jardine \\ SMF Fracture and Shock Physics Group, Cavendish Laboratory, University of Cambridge, CB3 OHE, \\ United Kingdom
}

\author{
Annette Glauser, Sara French and Steve Wortley
}

AWE plc, Aldermaston, RG7 4PR, United Kingdom

(Received 1 July 2015)

*Corresponding author. email: smw14@cam.ac.uk

Time resolved and integrated diagnostics including high speed photography, mass and optical spectroscopy, and optical-radiometry were used to study the impact response of high explosives in substantially more detail than possible with conventional sensitiveness tests. Specifically, we compare pentaerythritol tetranitrate (PETN) and cyclotetramethylene tetranitramine (HMX), with 2,6-diamino3,5-dinitropyrazine-1-oxide (LLM-105), the latter of which is currently receiving a great deal of interest as a promising insensitive high explosive. We conclude that under modest impact conditions, LLM-105 can undergo a low-level reaction whose characteristics explain apparently contradictory sensitiveness results obtained using standard tests. Our results demonstrate how more sophisticated diagnostics with large dynamic ranges, can quantify the hazard response of energetic materials in detail, enabling complex behaviour to be distinguished.

\section{Introduction}

In general, ignition of explosives by impact is a response with many integrated inputs; friction, jetting, viscous flow and adiabatic compression of trapped gas can all play a role ${ }^{1,2}$. Even for a given explosive, impact sensitiveness can be modified by physical characteristics such as grain-size, surface texture, and

internal perfection ${ }^{3}$. Despite the underlying complexity, standard machines and methodologies currently used to test sensitiveness typically reduce the results to a binary outcome: either a reaction, or lack of reaction, often referred to as 'go' or 'no-go'. The threshold condition is often subjective (operator notes spark or flash ${ }^{4}$ ), although quantitative measurements can also be used (sound level ${ }^{5}$ or the volume of evolved gas, as in the Rotter test ${ }^{6}$ ). In practice such instruments are usually only used to compare a ranking of sensitiveness between materials ${ }^{7,8}$.

In this paper, we present experiments in which a range of diagnostic instrumentation were combined to allow the impact response of explosives to be studied in considerably more quantitative detail than previously reported. We have applied the method to examine conventional explosives as well as the recently developed insensitive explosive 2,6-diamino-3,5-dinitropyrazine-1-oxide (LLM-105). In the case of LLM-105, we have observed a low-level reaction which explains apparently contradictory sensitiveness values obtained using different 'standard' tests. Our results highlight the importance of sophisticated diagnostics in obtaining a true representation of an explosive's response to impact, which is an important aspect of the safety characterisation of energetic materials.

LLM-105 is a relatively new energetic material first reported in the mid-1990s ${ }^{9}, 10$ that is currently receiving interest as a promising insensitive high explosive (IHE) ${ }^{11-18}$, i.e. an explosive which is inert to 
accidental or malicious stimuli. However, reports of its impact sensitiveness remain unclear. Although some batch-to-batch variation may have been present, test methods based on acoustic or light emission have suggested it is less sensitive than the standard explosive cyclotrimethylene trinitramine (RDX) ${ }^{10,12}$, ${ }^{19-21}$, whereas the Rotter test, which is based upon the volume of gas evolved upon reaction, suggests it is more sensitive than RDX. As measured in the Rotter test, LLM-105 has a Figure of Insensitivity (FoI) of $55 \pm 10$ whereas by definition RDX has an FOI of 80 (the lower the FoI the greater the sensitivity to impact). In this paper, we compare the behaviour of LLM-105 to the conventional high explosives pentaerythritol tetranitrate (PETN) and cyclotetramethylene tetranitramine (HMX), illustrated in Figure 1 .

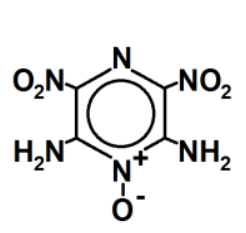

(a)

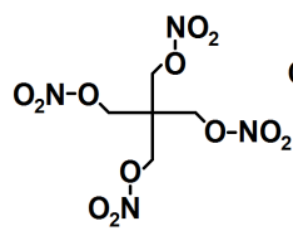

(b)

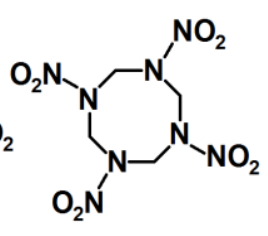

(c)

FIG. 1. Molecular structures of (a) LLM-105, (b) PETN and (c) HMX, showing the high density of nitro and amino groups.

Our experiments were based on performing impacts using the Cavendish Laboratory 'glass anvil drop-weight' apparatus ${ }^{22,23}$. The operation of the instrument is distinguished from standard impact sensitiveness tests ${ }^{24}$ as it does not generally seek to assess a threshold condition (such as the drop-height corresponding to $50 \%$ reaction probability), but rather to gain insight into the ignition mechanism. Essentially, the equipment consists of a $5.3 \mathrm{~kg}$ falling weight in which a toughened glass anvil is incorporated. The drop-weight is released from heights of up to $1.4 \mathrm{~m}$. At maximum height, the impact speed is around $5.4 \mathrm{~ms}^{-1}$. The key feature, shown schematically in Figure 2, is a periscopic lighting arrangement, which enables the impact event to be imaged on-axis using a high speed camera.

Initial experiments were performed in the 'classic' arrangement ${ }^{22}$ with a single lower glass anvil upon which a sample of loose-packed explosive powder was arranged (approx. $25 \mathrm{mg}, 5 \mathrm{~mm}$ diameter, $1 \mathrm{~mm}$ thick). Back-illumination using a diffused quartz-halogen lamp was adjusted to a brightness slightly lower than that expected from the sample during reaction. $128 \times 128$ pixel high speed video was recorded using a Phantom v7.1 camera (Vision Research) set to an inter-frame time of $15 \mu \mathrm{s}$, with an exposure time of $2 \mu \mathrm{s}$. However, during the course of this study, the apparatus was modified as shown in Figure 2. In the modified apparatus, gas-tight seals around the lower two toughened glass anvils enabled any gas evolved during reaction to be sampled, while simultaneously maintaining the optical path for visual diagnostics. The gas flowed over the sample in these experiments was drawn from atmosphere. 

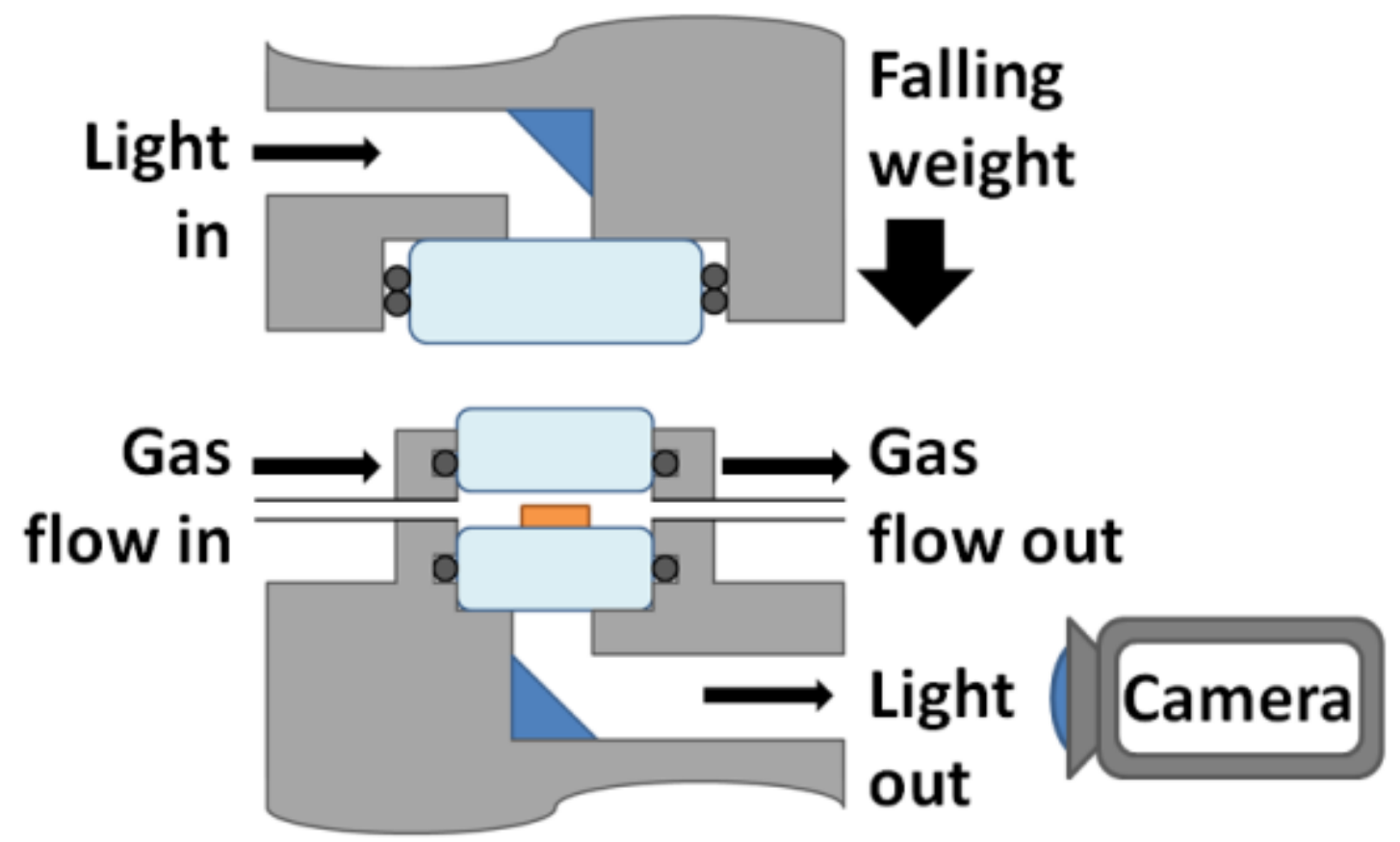

FIG. 2. Schematic of sample region within glass-anvil drop-weight apparatus. The falling weight contains a toughened glass striker that impacts a fixed stack of similarly toughened anvils with a sample at their centre. A periscopic lighting arrangement enables back-illumination and optical access to monitor reaction. Gas-tight seals and purging enable sampling of reaction products.

\section{Results and discussion}

Figure 3 shows typical high speed photographic sequences of reaction for samples of the well-known secondary explosives HMX and PETN (rows 1 and 2), which are compared to LLM-105 (row 3). The first frame in each sequence shows silhouettes of the uncompacted, unreacted samples. As each sample is compressed, the material expands radially, often undergoing abrupt jetting events as localised strain energy is released. For HMX and PETN the subsequent reaction phenomenology is relatively well known $^{22}$; reaction starts at one or two hotspots ${ }^{1,23,25}$, followed by growth of a self-illuminating reaction front, which quickly spreads out and consumes the sample. The reaction rates and light levels are similar for these two explosives, although PETN undergoes a phase transition under compression (widely attributed to pressure melting) becoming optically translucent shortly before the onset of reaction. 

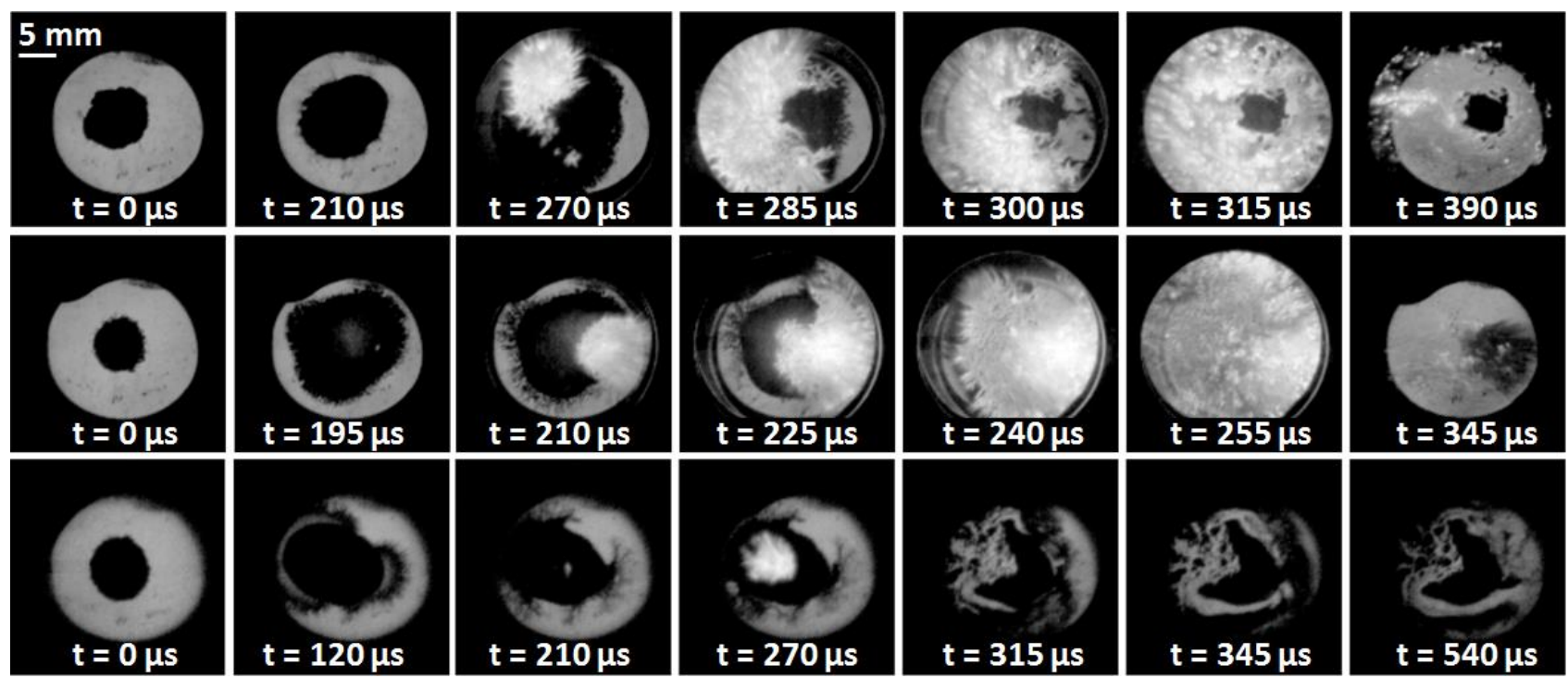

FIG. 3. High-speed photographic sequences of ignition by impact of three powdered explosive samples. Top row HMX; middle row PETN; bottom row LLM-105. Each frame exposure is $2 \mu \mathrm{s}$. Back-lighting was adjusted to match the approximate reaction light levels. The times indicated are relative to the onset of sample compression and the final frame in each row corresponds to the end of reaction activity. The PETN sample was totally consumed while the majority of the LLM-105 sample remained at the end of the test. The behaviour of the HMX sample was intermediate. The light of reaction from LLM-105 was approximately $1 \%$ of that from either HMX or PETN. The images were captured using a Phantom v7.1 camera (Vision Research).

We performed 65 experiments on LLM-105 and in the majority of cases only inert deformation and subsequent fracture of the consolidated material was observed. However, occasionally (approx. 10 times) we saw qualitatively different behaviour in which a bubble of gases appeared to burst through the perimeter of the sample. The gases then jetted around the circumference of the sample, meeting at a point diametrically opposite the breakout point. By reducing the backlighting and increasing the camera lens aperture, it was possible to determine that such LLM-105 events were self-illuminating, strongly suggesting reaction. In comparison to HMX or PETN, the self-luminance was dim and there appeared to be comparatively little consumption of material. The ratio of camera lens apertures required to achieve comparable light intensities between LLM-105 and HMX or PETN (allowing for statistical variation) was 91 ( $f / 1.2$ rather than $f / 11$, for the same $2 \mu$ s exposure), indicating an event which is approximately 100 times less bright. Examination of the glass anvils after the experiments showed that PETN and HMX samples caused widespread surface damage consisting of delamination of the outer glass layer forming $\sim 100 \mu \mathrm{m}$ 'flakes'. In the case of LLM-105 similar anvil damage was also observed, but was much more localised, consistent with weak propagation of reaction after ignition.

Overall, the photographic records along with an absence of the usual levels of light output or sound strongly suggest low-level reaction in LLM-105. There was no noticeable difference in impact noise between experiments on LLM-105, whereas for PETN or HMX a 'go' event can be distinctly heard by the operator. In particular, for LLM-105 the reaction is not sustained and a majority of the sample is left 
unconsumed. Such an event would lead to inconsistent results in conventional impact sensitiveness tests as production of gas will lead to a high apparent sensitiveness in a Rotter test ${ }^{6}$, whereas in the BAM Fall-hammer or ERL Drop-hammer tests ${ }^{4}$, a lack of light or sound will result in a low sensitiveness value.

To definitively confirm whether chemical reaction was occurring, mass-spectroscopy was performed on gases produced during the impact experiments by sampling from the flow ports around the lower anvils as shown in Figure 2. This was performed in conjunction with high-speed photography. To avoid atmospheric pressure forcing the anvils together (the space between the anvils is connected to a mass spectrometer which is under vacuum) statically compressing the samples and thereby changing their impact ignition characteristics, the enclosure between the anvils was maintained at near atmospheric pressure using a flow of bottled air as a purge gas. The purge gas, along with any entrained reaction products, then entered a quadrupole mass analyser (Hiden Analytical HAL 301) via a two-stage differential pumping arrangement. Note that the sampling rate was sufficiently slow that the measurement effectively became a time-integrated diagnostic, where the rise and fall times are dominated by the pumping characteristics of the system. The expected primary gas reaction products were $\mathrm{N}_{2}, \mathrm{H}_{2} \mathrm{O}$, and $\mathrm{CO}$, along with a variety of secondary species, including $\mathrm{CO}_{2}, \mathrm{NO}, \mathrm{N}_{2} \mathrm{O}, \mathrm{CH}_{2} \mathrm{O}$ and others. Since $\mathrm{N}_{2}$ and $\mathrm{H}_{2} \mathrm{O}$ were present in the purge gas, reaction was monitored using the other components. The known concentration of atmospheric argon (40 amu) provides absolute calibration. Figure 4 shows typical time resolved mass-traces for experiments where self-illuminating events were observed in the photographic record. An abrupt rise in the masses 30 and 44 was seen at the time of impact, as is to be expected for HMX and PETN. A similar rise in the case of LLM-105 confirms chemical reaction, and hence the origin of the light and apparent gas generation and flow previously observed. In experiments where high speed photography suggested an inert response, no sign of reaction in the mass-peaks was observed, thus demonstrating that the approach provides a consistent and clear distinction between 'go' and 'no-go' events. The ratio of mass-peak amplitudes varied noticeably between HMX, PETN and LLM-105. Future analysis of the reaction products may provide insight into reaction pathways during ignition and growth, compared with, for example, steady state detonation.

A gated optical spectrometer (EG\&G Princeton Applied Research 1471A) was used to examine the wavelengths present. With backlighting turned off, light produced by reaction was collected using an optical fibre. With the exposure time set to $2 \mathrm{~ms}$, the signal was integrated over the duration of the impact event. For HMX and PETN the majority of intensity was in a broad spectral peak at $590 \mathrm{~nm}$, associated with the de-excitation of nitrogen following formation of $\mathrm{N}_{2}$ from free radicals (the LewisRayleigh afterglow phenomenon ${ }^{26}$ ). For the experimental arrangement we used, the light emitted from the LLM-105 reaction processes was not bright enough to produce a useful spectrum and is not considered further here, but we note that this is a key point of interest for future research. 


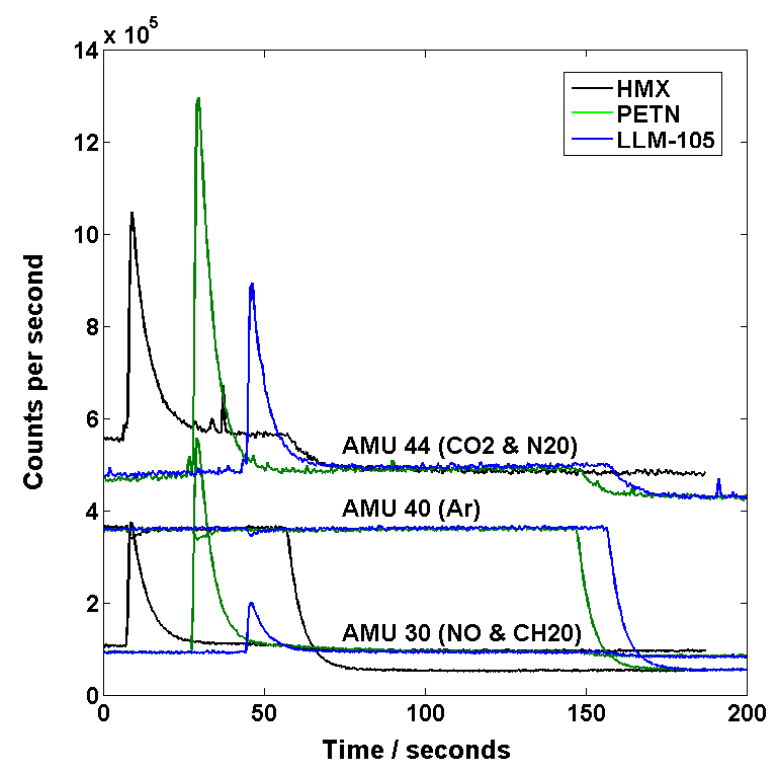

FIG. 4. Mass spectroscopy of gaseous reaction products from impact experiments with ignition. Traces have been time-shifted for clarity. Mass numbers were selected on the basis of expected ionised secondary products of reaction. The known level of atmospheric Ar provides absolute calibration (the mass-40 signal falls when the gas flow is closed after the experiment). Mass peaks were only ever observed in combination with self-luminous positive events observed using high speed photography and clearly demonstrate chemical reaction.

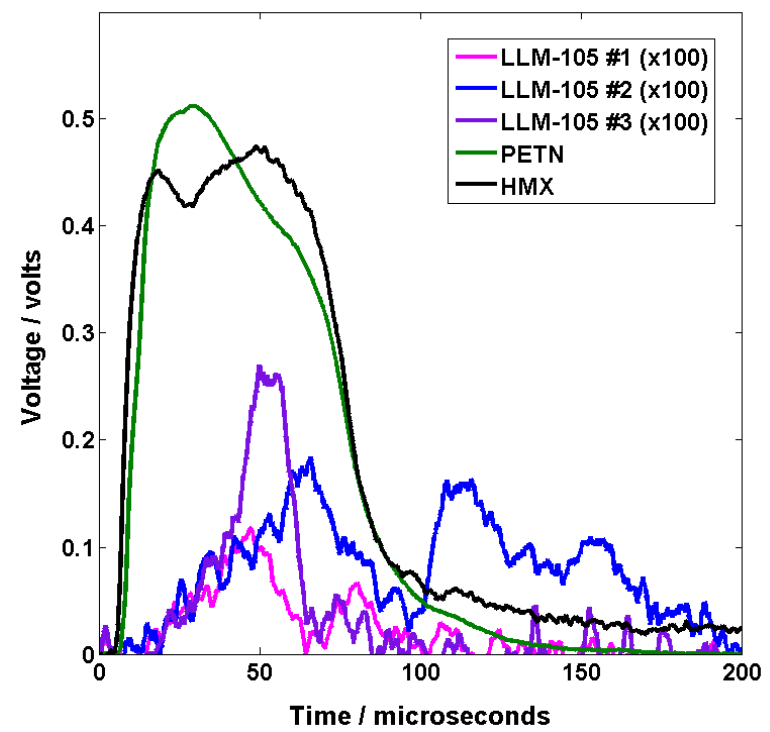

FIG. 5. Photodiode traces of the light emitted following ignition by impact. HMX and PETN show a sharp rise and a subsequent bright period of duration $\sim 70 \mu \mathrm{s}$, while LLM-105 rises more slowly and can last significantly longer. Note the LLM-105 signals have been multiplied by 100 to make the traces visible. 
To examine the intensity-time profile of the light produced, a photodiode detector (Centron model BXP65) was positioned on the lower surface of the bottom glass anvil and backlighting was turned off. The photodiode is an inexpensive method to quantify light output which can respond much more quickly than the high-speed camera. Several photodiode traces for LLM-105 reaction are compared in Figure 5 with typical responses for HMX and PETN. Both PETN and HMX exhibit sharp rises in intensity over a few microseconds, followed by a bright period of $\sim 70 \mu \mathrm{s}$. By contrast, the light intensities from LLM105 are roughly 100 times lower (consistent with the photographic record), rise much more slowly and can last considerably longer (around 100-200 $\mu \mathrm{s}$ ). The LLM-105 traces are also noticeably much less uniform, which we attribute to localised, irregular, propagation after ignition. More generally, the photodiode record is valuable as its large dynamic range enables both bright and dim events to be identified (e.g. by setting a 'go' threshold to compare with the intensity measurement) and quantified (by measuring duration and intensity).

\section{Conclusions}

Taken together, our results demonstrate low-level reaction in impacted samples of LLM-105, consisting of hotspot ignition followed by weak and erratic localised propagation, with comparatively little light output, but substantial levels of gas-production. These observations reconcile and explain previous, apparently contradictory, sensitiveness assessments for LLM-105. While the data presented does not provide insight into the precise reaction pathways, it does demonstrate characteristic behaviour. Establishing typical levels of light and gas production, as well as the reaction duration, will impose significant constraints on future models of ignition and growth. The observation of a reaction that cannot be correctly distinguished by standard sensitiveness tests also raises the question of the generality of the phenomenon: is this a unique characteristic of LLM-105 (or even a particular batch, grain size or morphology), or might similar low-level reactions have been missed in sensitiveness tests on other materials?

More generally, despite many decades of testing and development, existing sensitiveness tests have remained stubbornly subjective ${ }^{23}$. It is now clear that the current standardized assessment criteria are insufficient to describe the range of behaviour present, and need to be combined with more sophisticated diagnostics, such as those reported here. Together, these will enable clear characterization of the nature, intensity and duration of reaction, and suggest a route toward a more integrated and complete quantitative assessment of energetic sensitiveness.

\section{Acknowledgement}

The authors wish to acknowledge the funding and provision of samples for this research by AWE plc.

\section{References}

1. J. E. Field, N. K. Bourne, S. J. P. Palmer and S. M. Walley, Phil. Trans. R. Soc. Lond. A, 1992, 339, 269-283.

2. Y. Q. Wu, F. L. Huang and Z. Y. Zhang, RSC Advances, 2012, 2, 4152-4163.

3. S. M. Walley, J. E. Field and M. W. Greenaway, Mater. Sci. Technol., 2006, 22, 402-413. 
4. F. Trimborn, Explosivstoffe, 1970, 18, 49-56.

5. $\quad$ L. R. Simpson and M. F. Foltz, LLNL Small-Scale Drop-Hammer Impact Sensitivity Test, Report UCRL-ID-119665, Los Alamos National Laboratory, Los Alamos, NM, 1995.

6. H. N. Mortlock and J. Wilby, Explosivstoffe, 1966, 14, 49-55.

7. L. C. Smith, in Proc. Conf. on the Standardization of Safety and Performance Tests for Energetic Materials. Vol. 1, eds. L. Avrami, H. J. Matsugama and R. F. Walker, US Army Armament

Research and Development Command, Dover, NJ, 1977, pp. 397-414.

8. K. R. Becker and R. W. Watson, in Proc. Conf. on the Standardization of Safety and Performance Tests for Energetic Materials. Vol. 1, eds. L. Avrami, H. J. Matsugama and R. F. Walker, US Army Armament Research and Development Command, Dover, NJ, 1977, pp. 415430.

9. R. A. Hollins, R. A. Nissan, W. S. Wilson and R. D. Gilardi, 2,6-Diamino-3,5-Dinitropyrazine1-Oxide: A new Insensitive Explosive, Report NAWCWPNS TP 8228, Naval Air Warfare Center Weapons Division, China Lake, CA, 1995.

10. P. Pagoria, A. Mitchell, R. Schmidt, R. Simpson, F. Garcia, J. Forbes, J. Cutting, R. Lee, R. Swansiger and D. M. Hoffman, in Proc. Insensitive Munitions \& Energetic Materials Technology Symposium, National Defense Industrial Association, Lancaster, PA, 1998, paper I3.

11. F. Garcia, K. S. Vandersall, C. M. Tarver and P. A. Urtiew, AIP Conf. Proc., 2007, 955, $907-$ 910.

12. R. K. Weese, A. K. Burnham, H. C. Turner and T. D. Tran, J. Therm. Anal. Calorimetry, 2007, 89, 465-473.

13. J. C. Gump, C. A. Stoltz, B. P. Mason, B. G. Freedman, J. R. Ball and S. M. Peiris, J. Appl. Phys., 2011, 110, 073523.

14. C. W. An, H. Q. Li, X. H. Geng, J. L. Li and J. Y. Wang, Propell. Explos. Pyrotech., 2013, 38, 172-175.

15. W. Z. Xu, C. W. An, J. Y. Wang, J. Dong and X. H. Geng, Propell. Explos. Pyrotech., 2013, 38, 136-141.

16. J. Zhang, P. Wu, Z. J. Yang, B. Gao, J. H. Zhang, P. Wang, F. D. Nie and L. Y. Liao, Propell. Explos. Pyrotech., 2014, 39, 653-657.

17. S. Kucheyev, A. E. Gash, P. F. Pagoria, K. R. Lorenz, D. am Ende, S. Amato, L. J. Ping, S. Rothstein and J. Salan, in Proc. 15th International Detonation Symposium, eds. J. Carney and J. Maienschein, Office of Naval Research, Arlington, VA, 2015, pp. 567-575.

18. P. Vitello, K. T. Lorenz, D. M. Hoffman, L. E. Fried and P. C. Souers, in Proc. 15th International Detonation Symposium, eds. J. Carney and J. Maienschein, Office of Naval Research, Arlington, VA, 2015, pp. 1373-1380.

19. P. Pagoria, A. R. Mitchell, R. D. Schmidt, R. L. Simpson, F. Garcia, J. W. Forbes, R. W. Swansiger and D. M. Hoffman, Synthesis, Scale-up and Characterization of 2,6-Diamino-3,5Dinitropyrazine-1-Oxide (LLM-105), Report UCRL-JC-130518, Lawrence Livermore National Laboratory, Livermore, CA, 1998.

20. T. D. Tran, P. F. Pagoria, D. M. Hoffman, B. Cunningham, R. L. Simpson, R. S. Lee and J. L. Cutting, in Proc. Twelth International Detonation Symposium, Office of Naval Research, Arlington, VA, 2002, pp. 440-447.

21. D. M. Hoffman, K. T. Lorenz, B. Cunningham and F. Gagliardi, in Proc. 39th Int. Ann. Conf. of the Fraunhofer Institut für Chemische Technologie, Pfintztal, Germany, 2008, paper 29.

22. S. N. Heavens and J. E. Field, Proc. R. Soc. Lond. A, 1974, 338, 77-93. 
23. S. M. Walley, J. E. Field, R. A. Biers, W. G. Proud, D. M. Williamson and A. P. Jardine, Propell. Explos. Pyrotech., 2015, 40, 351-365.

24. Y. S. Wen, X. P. Long, Y. Xiang, S. J. Yu, X. G. Dai and M. Li, Propell. Explos. Pyrotech., 2015, 40, 433-438.

25. J. E. Field, Accounts Chem. Res., 1992, 25, 489-496.

26. U. H. Kurzweg and H. P. Broida, J. Molec. Spectrosc., 1959, 3, 388-404.

AWE @ Crown Owned copyright (2016) 